\title{
Chinese herbal medicine reduces mortality in patients with severe and critical coronavirus disease 2019: a retrospective cohort study
}

Guohua Chen ${ }^{1, *}$, Wen $\mathrm{Su}^{1, *}$, Jiayao Yang ${ }^{1, *}$, Dan Luo ${ }^{1, *}$, Ping Xia ${ }^{1}$, Wen $\mathrm{Jia}^{1}$, Xiuyang $\mathrm{Li}^{2}$, Chuan Wang ${ }^{1}$, Suping Lang ${ }^{3}$, Qingbin Meng ${ }^{1}$, Ying Zhang ${ }^{4}$, Yuhe $\mathrm{Ke}^{1}$, An Fan ${ }^{3}$, Shuo Yang ${ }^{1}$, Yujiao Zheng ${ }^{2}$, Xuepeng Fan ${ }^{1}$, Jie Qiao

Fengmei Lian $(\bowtie)^{2}$, Li Wei $(\bowtie)^{1}$, Xiaolin Tong $(\bowtie)^{2}$

${ }^{1}$ Wuhan No.1 Hospital (Wuhan Integrated TCM \& Western Medicine Hospital), Wuhan 430022, China; ${ }^{2}$ Guang'anmen Hospital, China Academy of Chinese Medical Sciences, Beijing 100053, China; ${ }^{3}$ GCP ClinPlus Co., Ltd., Beijing 100160, China; ${ }^{4}$ Beijing University of Chinese Medicine, Beijing 100029, China; ${ }^{5}$ Hubei University of Traditional Chinese Medicine, Wuhan 430060, China

(C) Higher Education Press 2020

\begin{abstract}
This study aimed to evaluate the efficacy of Chinese herbal medicine (CHM) in patients with severe/ critical coronavirus disease 2019 (COVID-19). In this retrospective study, data were collected from 662 patients with severe/critical COVID-19 who were admitted to a designated hospital to treat patients with severe COVID-19 in Wuhan before March 20, 2020. All patients were divided into an exposed group (CHM users) and a control group (non-users). After propensity score matching in a 1:1 ratio, $156 \mathrm{CHM}$ users were matched by propensity score to 156 non-users. No significant differences in seven baseline clinical variables were found between the two groups of patients. All-cause mortality was reported in 13 CHM users who died and 36 non-users who died. After multivariate adjustment, the mortality risk of CHM users was reduced by $82.2 \%$ (odds ratio $0.178,95 \%$ CI 0.076 0.418; $P<0.001)$ compared with the non-users. Secondly, age (odds ratio $1.053,95 \%$ CI $1.023-1.084 ; P<0.001)$ and the proportion of severe/critical patients (odds ratio $0.063,95 \%$ CI $0.028-0.143 ; P<0.001$ ) were the risk factors of mortality. These results show that the use of CHM may reduce the mortality of patients with severe/ critical COVID-19.
\end{abstract}

Keywords COVID-19; CHM; mortality; a retrospective cohort study

\section{Introduction}

In December 2019, a breakout of coronavirus disease 2019 (COVID-19) was reported. The World Health Organization (WHO) has declared COVID-19 as a global pandemic [1]. At present, the number of death cases from COVID-19 is increasing rapidly, and its mortality rate is significantly higher than that of influenza [2]. By March 29, 2020, the total number of confirmed cases worldwide was 634835 , and the number of deaths was as high as 29891 [3]. No vaccine or antiviral medicine has confirmed clinical effect

Received April 14, 2020; accepted July 8, 2020

Correspondence: Fengmei Lian, 1fm565@sohu.com;

Li Wei, y077088@sina.com;

Xiaolin Tong, tongxiaolin@vip.163.com

${ }^{*}$ These authors contributed equally to this work. to prevent or treat COVID-19 currently, which has brought great difficulties to the prevention and control of the epidemic [4]. A recent clinical study has reported that the antiviral drugs lopinavir and ritonavir have no clinical benefit in the treatment of patients with severe COVID-19 [5].

China has constantly emphasized on the use of Chinese and Western medicine in the fight against COVID-19. Chinese herbal medicine (CHM), which is the main component of complementary and alternative medicine, plays an important role in the whole process. In the fifth edition of the Diagnosis and Treatment Guideline for COVID-19 (Trial Version) released by the National Health Commission of the People's Republic of China, CHM treatment has been included. Clinical effects of CHM or integrated Chinese and Western medicine treatments on patients with mild COVID-19 have been recently reported [6]. However, no studies have reported the efficacy 
evaluation and clinical outcomes of CHM intervention in patients with severe COVID-19. The present study aimed to analyze the influence of demographics, clinical treatment, laboratory test, and other factors on the mortality of patients with severe/critical COVID-19 and to evaluate the effect of CHM in reducing the mortality of these patients.

\section{Materials and methods}

\section{Study design and participants}

We performed a single-center, retrospective cohort study in a hospital in Wuhan, China, which is one of the largest designated hospitals to treat patients with severe COVID19 by using integrated Chinese and Western medicine. We retrospectively analyzed patients with severe/critical COVID-19 who were admitted to hospital and had clinical outcomes before March 20, 2020. The patients were diagnosed and typed in accordance with the fifth edition of the Diagnosis and Treatment Guideline for COVID-19 (Trial Version) [7]. Severe cases in accordance with the following criteria were included: (1) respiratory distress $(\geqslant 30$ breaths $/ \mathrm{min}$ ); (2) oxygen saturation $\leqslant 93 \%$ at rest; (3) arterial partial pressure of oxygen $\left(\mathrm{PaO}_{2}\right) /$ fraction of inspired oxygen $\left(\mathrm{FiO}_{2}\right) \leqslant 300 \mathrm{mmHg}(1 \mathrm{mmHg}=$ $0.133 \mathrm{kPa}$ ). Cases with chest imaging showing obvious lesion progression within 24-48 $\mathrm{h}>50 \%$ were managed as severe cases. Critical cases in accordance with the following criteria were included: (1) respiratory failure and requiring mechanical ventilation; (2) shock; (3) with other organ failure that requires intensive care unit care. The clinical outcome was discharge or death, and the criteria for hospital discharge were absence of fever for at least $72 \mathrm{~h}$, substantial improvement in both lungs in chest computed tomography (CT), clinical remission of respiratory symptoms, and two throat-swab samples negative for SARS-CoV-2 RNA obtained at least $24 \mathrm{~h}$ apart.

This study was approved by the institutional ethics board of Wuhan Integrated TCM and Western Medicine Hospital (No. [2020]8) and was registered with chictr.org. cn (ChiCTR2000030719). Due to the retrospective nature of the study, the requirement of informed consent was waived.

\section{Data collection}

Data for the study were obtained from the HIS electronic medical records in the hospital, including demographics, clinical symptoms, laboratory examinations, treatment measures, and outcomes. Two researchers from the hospital manually collected and examined the data, and a dispute between the first two researchers was settled by a third researcher.

\section{Procedures}

Patients were treated with oxygen, antivirals (such as interferon or ribavirin), antibiotics (such as moxifloxacin, cefoperazone sodium, and sulbactam sodium), and Chinese medicine based on the Diagnosis and Treatment Guideline for COVID-19 (Trial Version). Critical patients were treated with noninvasive mechanical ventilation, invasive mechanical ventilation, and extracorporeal membrane oxygenation to support life. All CHM treatments were administered in accordance with the traditional Chinese medicine (TCM) treatment of the abovementioned guideline. The CHM mainly consisted of Fuling (Poria cocos (Schw.) Wolf.), Huangqi (Astragalus membranaceus (Fisch.) Bge. var. mongholicus (Bge.) Hsiao.), Huoxiang (Pogostemon cablin (Blanco) Benth.), Kuxingren (Prunus armeniaca L. var. ansu Maxim.), Baizhu (Atractylodes macrocephala Koidz.), Banxia (Pinellia ternata (Thunb.) Breit.), Gancao (Glycyrrhiza uralensis Fisch.), Houpo (Magnolia officinalis Rehd. et Wils.), Mahuang (Ephedra sinica Stapf), Guizhi (Cinnamomum cassia Presl), Huangqin (Scutellaria baicalensis Georgi.), Sharen (Amomum villosum Lour.), Jiegeng (Platycodon grandiflorum (Jacq.) A.DC.), Peilan (Eupatorium fortunei Turcz.), and Dangsheng (Codonopsis pilosula (Franch.) Nannf.) purchased from Hubei Tianji TCM Decoction Pieces Co., Ltd. This TCM decoction "Mahuang Liu Jun Tang" was administered orally $200 \mathrm{~mL}$ each time, twice a day in hospital days.

The laboratory examinations were standardized in the laboratory department of the hospital. Throat-swab specimens were obtained for SARS-CoV-2 PCR examination at the time of admission and during hospitalization, and the SARS-CoV-2 nucleic acid detection kit (fluorescent PCR) was purchased from Shanghai Zhijiang Biotechnology Co., Ltd. Routine blood examinations included complete blood count, coagulation profile, and serum biochemical tests (including renal and liver function, creatine kinase, myocardial enzymogram, high-sensitivity troponin, electrolytes, humoral immune function, and C-reactive protein). CT scan was also performed for all inpatients. Frequency of examinations was determined by the treating physician.

\section{Outcomes}

The primary outcome was all-cause mortality. The considered factors were age, sex, disease duration, medical history, treatment measures (including oxygen, antivirals, antibiotics, Chinese patent medicine, and CHM). Cause of death was considered from the aspects of respiratory failure, septic shock, gastrointestinal bleeding, heart failure, stroke, multiple organ dysfunction syndrome (MODS), and surgery. 


\section{Propensity score}

Propensity score matching (PSM) was used. Three variables (age, sex, and disease stage) in the propensity score were chosen on the basis of clinical significance. Each patient who received CHM was matched to a patient who did not on propensity score by using the greedy nearest neighbor matching without replacement within a caliper. The caliper width was 0.05 of the logit-transformed propensity score. The absolute standardized difference was used to evaluate the balance of baseline characteristics between the two groups after matching, and all were checked to be less than 0.25 .

\section{Statistical analyses}

Continuous and categorical variables were presented as median (IQR) and $n(\%)$, respectively. The Mann-Whitney $\mathrm{U}$ test, $\chi^{2}$ test, or Fisher's exact test was performed to compare differences between CHM users and non-users where appropriate. Univariable and multivariable logistic regression models were performed to explore the risk factors associated with the mortality of patients with severe/critical COVID-19. PSM was used to balance the two groups considering the known confounders. Using the application of CHM as the dependent variable, we incorporated multiple clinically relevant covariates into our analysis as independent variables (all baseline characteristics). Statistical significance was considered at $P<0.05$. Statistical analyses were conducted using SAS version 9.4.

\section{Results}

\section{Demographic and patient characteristics}

By March 20, 2020, 1476 cases of COVID-19 patients were hospitalized in Wuhan Integrated TCM and Western Medicine Hospital. After excluding 170 transferred patients and 644 mild patients, we included 662 patients for the final analysis, including 484 CHM users and 178 non-users (Fig. 1). The 662 patients were composed of 562 $(84.9 \%)$ severe patients and $100(15.1 \%)$ critical patients. A total of $71(10.7 \%)$ patients died, and $591(89.3 \%)$ patients were discharged from the hospital. Of the 71 deaths, 49 were due to acute respiratory failure $(69.0 \%), 1$ $(1.4 \%)$ was due to respiratory failure with heart failure, and $1(1.4 \%)$ was due to respiratory failure with myocardial infarction. Respiratory failure with tumors occurred in 2 $(2.8 \%)$ cases, respiratory failure with septic shock in 4 $(5.6 \%)$ cases, septic shock in $5(7 \%)$ cases, MODS in 3 $(4.2 \%)$ cases, death after the operation in $2(2.8 \%)$

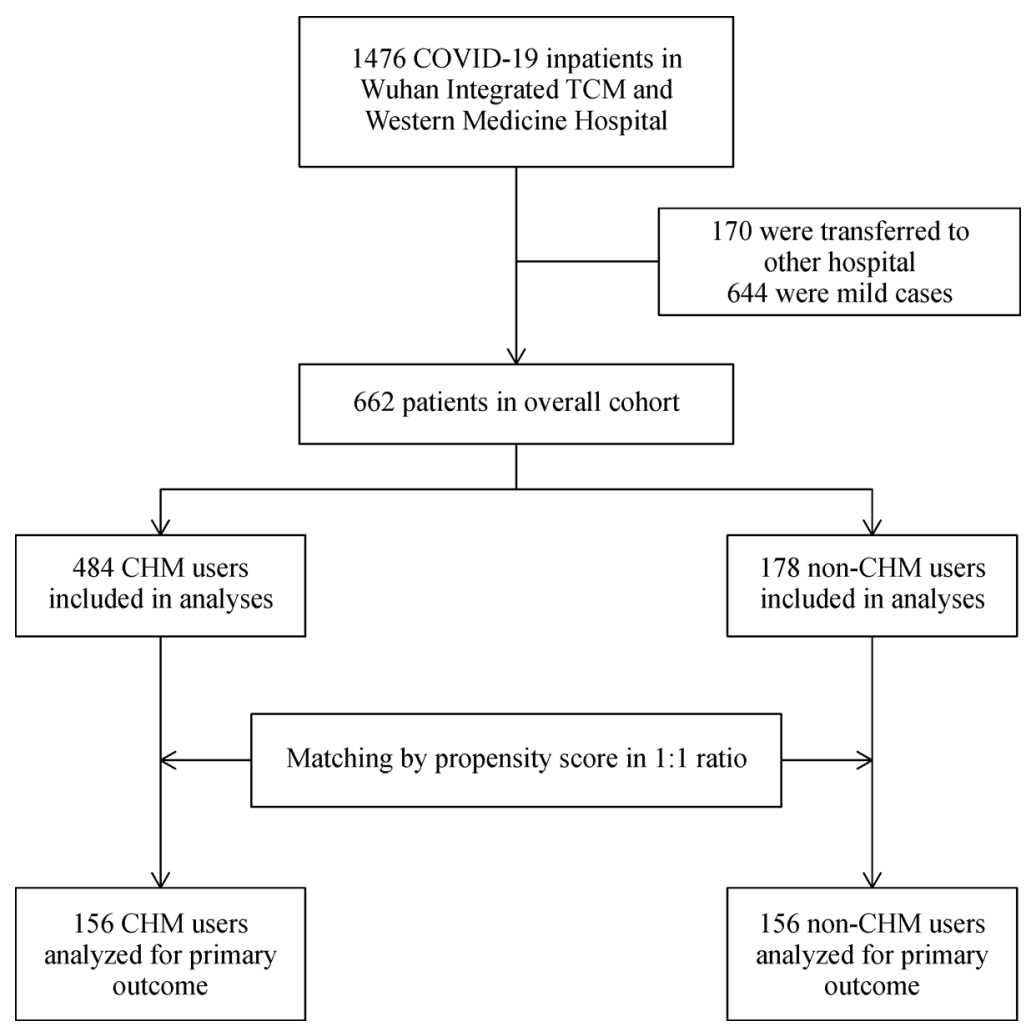

Fig. 1 Study profile. 
cases, gastrointestinal bleeding in $1(1.4 \%)$ case, acute myocardial infarction in $2(2.8 \%)$ cases, and stroke in 1 $(1.4 \%)$ case.

\section{Baseline characteristics before PSM}

In the group of CHM users, 444 patients $(91.7 \%)$ were severe and 40 patients $(8.3 \%)$ were critical. In the group of non-users, $118(66.3 \%)$ were severe and $60(33.7 \%)$ were critical. A total of $15(3.1 \%)$ CHM users died and 56 (31.5\%) non-users died. We analyzed clinical variables between the two groups. Differences in age, sex, medical history, antibiotics, hormone treatment, and oxygen therapy were found between the two groups. Differences in signs and symptoms, such as fever and constipation, were also detected. With respect to laboratory tests, differences were found in the levels of leukocytes, lymphocytes, monocytes, C-reactive protein, creatine kinase, and troponin-I between the two groups. In terms of treatment, the two groups showed significant differences in antibiotics, Chinese patent medicine, and oxygen therapy (Table 1).

Table 1 Baseline characteristics of study patients

\begin{tabular}{|c|c|c|c|c|}
\hline & Total $(n=662)$ & CHM users $(n=484)$ & CHM non-users $(n=178)$ & $P$ value \\
\hline$\overline{\text { Age (year) }}$ & $60(47,70)$ & $57(45,68)$ & $66(54,76)$ & $<0.001$ \\
\hline Sex & & & & 0.011 \\
\hline Men & $296(44.7 \%)$ & $202(41.7 \%)$ & $94(52.8 \%)$ & \\
\hline Women & $366(55.3 \%)$ & $282(58.3 \%)$ & $84(47.2 \%)$ & \\
\hline Smoking history & & & & 0.348 \\
\hline Never & $575(86.9 \%)$ & $417(86.2 \%)$ & $158(88.8 \%)$ & \\
\hline Ever & $15(2.3 \%)$ & $12(2.5 \%)$ & $3(1.7 \%)$ & \\
\hline Current & $20(3.0 \%)$ & $18(3.7 \%)$ & $2(1.1 \%)$ & \\
\hline Other & $52(7.9 \%)$ & $37(7.6 \%)$ & $15(8.4 \%)$ & \\
\hline Comorbidity & $354(53.5 \%)$ & $246(50.8 \%)$ & $108(60.7 \%)$ & 0.024 \\
\hline Chronic obstructive lung disease & $19(2.9 \%)$ & $10(2.1 \%)$ & $9(5.1 \%)$ & 0.041 \\
\hline Hypertension & $208(31.4 \%)$ & $147(30.4 \%)$ & $61(34.3 \%)$ & 0.338 \\
\hline Cardiovascular disease & $53(8.0 \%)$ & $40(8.3 \%)$ & $13(7.3 \%)$ & 0.686 \\
\hline Diabetes & $94(14.2 \%)$ & $69(14.3 \%)$ & $25(14.0 \%)$ & 0.945 \\
\hline Malignancy & $12(1.8 \%)$ & $7(1.4 \%)$ & $5(2.8 \%)$ & 0.321 \\
\hline Cerebrovascular disease & $38(5.7 \%)$ & $26(5.4 \%)$ & $12(6.7 \%)$ & 0.502 \\
\hline Chronic kidney disease & $3(0.6 \%)$ & $0(0.0 \%)$ & $3(0.5 \%)$ & 0.568 \\
\hline Chronic liver disease & $8(1.2 \%)$ & $5(1.0 \%)$ & $3(1.7 \%)$ & 0.449 \\
\hline Signs and symptoms & $658(99.4 \%)$ & $481(99.4 \%)$ & $177(99.4 \%)$ & 1.000 \\
\hline Fever & $450(68.0 \%)$ & $345(71.3 \%)$ & $105(59.0 \%)$ & 0.003 \\
\hline Dry cough & $408(61.6 \%)$ & $298(61.6 \%)$ & $110(61.8 \%)$ & 0.957 \\
\hline Expectoration & $184(27.8 \%)$ & $129(26.7 \%)$ & $55(30.9 \%)$ & 0.280 \\
\hline Fatigue & $298(45.0 \%)$ & $209(43.2 \%)$ & $89(50.0 \%)$ & 0.118 \\
\hline Shortness of breath & $221(33.4 \%)$ & $153(31.6 \%)$ & $68(38.2 \%)$ & 0.111 \\
\hline Nasal obstruction & $21(3.2 \%)$ & $18(3.7 \%)$ & $3(1.7 \%)$ & 0.186 \\
\hline Pharyngalgia & $39(5.9 \%)$ & $33(6.8 \%)$ & $6(3.4 \%)$ & 0.095 \\
\hline Hemoptysis & $4(0.6 \%)$ & $1(0.2 \%)$ & $3(1.7 \%)$ & 0.062 \\
\hline Chills & $46(6.9 \%)$ & $33(6.8 \%)$ & $13(7.3 \%)$ & 0.828 \\
\hline Headache & $45(6.8 \%)$ & $30(6.2 \%)$ & $15(8.4 \%)$ & 0.312 \\
\hline Myalgia & $74(11.2 \%)$ & $55(11.4 \%)$ & $19(10.7 \%)$ & 0.803 \\
\hline Vomiting & $35(5.3 \%)$ & $22(4.5 \%)$ & $13(7.3 \%)$ & 0.160 \\
\hline Diarrhea & $71(10.7 \%)$ & $55(11.4 \%)$ & $16(9.0 \%)$ & 0.381 \\
\hline Constipation & $12(1.8 \%)$ & $5(1.0 \%)$ & $7(3.9 \%)$ & 0.020 \\
\hline \multicolumn{5}{|l|}{ Laboratory tests } \\
\hline White blood cell count, $\times 10^{9} / \mathrm{L}$ & $5.39(4.30,6.87)$ & $5.46(4.42,7.00)$ & $5.02(3.83,6.60)$ & 0.020 \\
\hline Red blood cell count, $\times 10^{9} / \mathrm{L}$ & $4.200(3.860,4.590)$ & $4.205(3.870,4.610)$ & $4.150(3.810,4.560)$ & 0.314 \\
\hline Neutrophil count, $\times 10^{9} / \mathrm{L}$ & $3.200(2.380,4.460)$ & $3.260(2.430,4.530)$ & $3.010(2.210,4.000)$ & 0.089 \\
\hline Lymphocyte count, $\times 10^{9} / \mathrm{L}$ & $1.38(0.96,1.81)$ & $1.44(1.01,1.88)$ & $1.19(0.84,1.58)$ & $<0.001$ \\
\hline Monocyte count, $\times 10^{9} / \mathrm{L}$ & $0.51(0.37,0.66)$ & $0.52(0.39,0.68)$ & $0.46(0.34,0.61)$ & 0.002 \\
\hline Platelet count, $\times 10^{9} / \mathrm{L}$ & $208.00(161.00,274.00)$ & $211.00(166.00,277.00)$ & $202.00(149.00,260.00)$ & 0.093 \\
\hline
\end{tabular}




\begin{tabular}{|c|c|c|c|c|}
\hline & & & & (Continued $)$ \\
\hline & Total $(n=662)$ & CHM users $(n=484)$ & CHM non-users $(n=178)$ & $P$ value \\
\hline Hemoglobin, $g / L$ & $128.00(118.00,140.00)$ & $128.00(118.00,141.20)$ & $128.00(118.00,138.00)$ & 0.515 \\
\hline C-reactive protein, mg/L & $7.00(3.11,30.04)$ & $4.60(3.11,26.50)$ & $14.00(3.11,41.50)$ & $<0.001$ \\
\hline Albumin, $\mathrm{g} / \mathrm{L}$ & $35.70(32.35,39.00)$ & $35.70(32.50,39.00)$ & $35.50(32.20,38.60)$ & 0.102 \\
\hline Alanine aminotransferase, $\mathrm{U} / \mathrm{L}$ & $22(14,36)$ & $23(14,37)$ & $20(14,32)$ & 0.207 \\
\hline Aspartate aminotransferase, $\mathrm{U} / \mathrm{L}$ & $25(19,37)$ & $25(19,37)$ & $25(19,37)$ & 0.879 \\
\hline Blood urea nitrogen, $\mathrm{mmol} / \mathrm{L}$ & $4.10(3.30,5.30)$ & $4.10(3.30,5.30)$ & $4.00(3.20,5.40)$ & 0.703 \\
\hline Creatinine, $\mu \mathrm{mol} / \mathrm{L}$ & $63.0(54.0,77.4)$ & $63.0(54.0,77.0)$ & $62.0(54.0,79.4)$ & 0.937 \\
\hline Creatine kinase, U/L & $61.00(41.00,94.00)$ & $58.00(39.00,85.00)$ & $70.00(46.00,143.00)$ & 0.002 \\
\hline Lactate dehydrogenase, $\mathrm{U} / \mathrm{L}$ & $211.0(163.0,285.0)$ & $207.0(161.0,285.0)$ & $223.5(170.0,309.0)$ & 0.195 \\
\hline $\begin{array}{l}\text { High-sensitivity cardiac troponin I, } \\
\mathrm{pg} / \mathrm{mL}\end{array}$ & $0.007(0.002,0.034)$ & $0.006(0.002,0.020)$ & $0.012(0.004,2.900)$ & 0.004 \\
\hline $\mathrm{IgG}, \mathrm{g} / \mathrm{L}$ & $10.40(8.15,12.90)$ & $10.40(8.17,13.90)$ & $10.60(7.94,11.90)$ & 0.555 \\
\hline $\operatorname{IgA}, \mathrm{g} / \mathrm{L}$ & $2.19(1.56,2.94)$ & $2.27(1.83,3.12)$ & $2.05(1.36,2.51)$ & 0.071 \\
\hline IgM, g/L & $0.95(0.73,1.33)$ & $0.94(0.63,1.38)$ & $0.96(0.83,1.33)$ & 0.516 \\
\hline $\mathrm{C} 3, \mathrm{~g} / \mathrm{L}$ & $1.07(0.92,1.24)$ & $1.05(0.91,1.25)$ & $1.14(0.93,1.24)$ & 0.355 \\
\hline $\mathrm{C} 4, \mathrm{~g} / \mathrm{L}$ & $0.270(0.206,0.340)$ & $0.245(0.192,0.326)$ & $0.303(0.253,0.349)$ & 0.066 \\
\hline \multicolumn{5}{|l|}{ Imaging features } \\
\hline Consolidation & $522(78.9 \%)$ & $386(79.8 \%)$ & $136(76.4 \%)$ & 0.322 \\
\hline Pleural effusion & $28(4.2 \%)$ & $16(3.3 \%)$ & $12(6.7 \%)$ & 0.050 \\
\hline \multicolumn{5}{|l|}{ Treatment } \\
\hline Antibiotics & $516(77.9 \%)$ & $362(74.8 \%)$ & $154(86.5 \%)$ & 0.001 \\
\hline Antiviral treatment & $553(83.5 \%)$ & $407(84.1 \%)$ & $146(82.0 \%)$ & 0.525 \\
\hline Chinese patent medicine & $437(66.0 \%)$ & $331(68.4 \%)$ & $106(59.6 \%)$ & 0.033 \\
\hline Hormone & $149(22.5 \%)$ & $95(19.6 \%)$ & $54(30.3 \%)$ & 0.003 \\
\hline Oxygen therapy & & & & $<0.001$ \\
\hline Mask oxygen inhalation & $454(68.6 \%)$ & $361(74.6 \%)$ & $93(52.2 \%)$ & \\
\hline Nasal tube of oxygen inhalation & $161(24.3 \%)$ & $107(22.1 \%)$ & $54(30.3 \%)$ & \\
\hline Noninvasive mechanical ventilation & $45(6.8 \%)$ & $16(3.3 \%)$ & $29(16.3 \%)$ & \\
\hline Invasive mechanical ventilation & $2(0.3 \%)$ & $0(0.0 \%)$ & $2(1.1 \%)$ & \\
\hline
\end{tabular}

\section{Baseline characteristics and all-cause mortality after PSM}

To balance the differences in baseline characteristics and reduce confounding factors, we performed PSM by incorporating age, sex, and disease severity as independent variables. After PSM in a 1:1 ratio, the CHM users and non-users were both 156. The two groups showed differences in terms of medical history, antibiotics, and hormone treatment at baseline (Table 2). The mortality rates of the $\mathrm{CHM}$ and non-CHM groups were $8.3 \%$ and $23.1 \%$, respectively.

Univariate logistic regression analysis of the death outcomes of the two groups revealed significant differences in the effects of CHM, age, and disease severity on the mortality rate, and multivariate logistic regression analysis showed significant differences in the effects of CHM, age, and medical history on the mortality rate (Table 3). The risk of death in the CHM group reduced by $88.1 \%$ compared with that in the non-CHM group. Age was also one of the important factors influencing the mortality rate. For every 1 year of age increase, the risk of death increased by $5.0 \%$.

\section{Discussion}

Since the outbreak of COVID-19, the rising mortality has caused widespread concern. Existing research results suggest that male, age, and organ failure severity are the risk factors of death in patients with COVID-19 $[8,9]$. Our study has confirmed that age is an important factor affecting mortality in patients with COVID-19, which is consistent with the results of previous studies [10]. In the fifth edition of the Diagnosis and Treatment Guideline for COVID-19 (Trial Version) released by China, patients with severe and critical COVID-19 were classified based on whether respiratory failure or other organ failure occurred. In this study, disease severity was relevant to organ failure severity, which also validated the results of previous studies that organ failure severity is an important risk factor affecting mortality. 
Table 2 Baseline characteristics of study patients after PSM

\begin{tabular}{|c|c|c|c|c|}
\hline & Total $(n=312)$ & CHM users $(n=156)$ & CHM non-users $(n=156)$ & $P$ value \\
\hline Age (year) & $63(51,72)$ & $63(54,72)$ & $63(49,73)$ & 0.973 \\
\hline Sex & & & & 0.495 \\
\hline Men & $142(45.5 \%)$ & $68(43.6 \%)$ & $74(47.4 \%)$ & \\
\hline Women & $170(54.5 \%)$ & $88(56.4 \%)$ & $82(52.6 \%)$ & \\
\hline The stage of disease & & & & 1.000 \\
\hline Severe cases & $236(75.6 \%)$ & $118(75.6 \%)$ & $118(75.6 \%)$ & \\
\hline Critical cases & $76(24.4 \%)$ & $38(24.4 \%)$ & $38(24.4 \%)$ & \\
\hline Comorbidity & $174(55.8 \%)$ & $77(49.4 \%)$ & $97(62.2 \%)$ & 0.023 \\
\hline Imaging features & $287(92.0 \%)$ & $147(94.2 \%)$ & $140(89.7 \%)$ & 0.144 \\
\hline \multicolumn{5}{|l|}{ Treatment } \\
\hline Antibiotics & $251(80.4 \%)$ & $116(74.4 \%)$ & $135(86.5 \%)$ & 0.007 \\
\hline Antiviral treatment & $266(85.3 \%)$ & $136(87.2 \%)$ & $130(83.3 \%)$ & 0.338 \\
\hline Chinese patent medicine & $200(64.1 \%)$ & $107(68.6 \%)$ & $93(59.6 \%)$ & 0.098 \\
\hline Hormone & $84(26.9 \%)$ & $33(21.2 \%)$ & $51(32.7 \%)$ & 0.022 \\
\hline Oxygen therapy & & & & 0.657 \\
\hline Mask oxygen inhalation & $184(59.0 \%)$ & $95(60.9 \%)$ & $89(57.1 \%)$ & \\
\hline Nasal tube of oxygen inhalation & $95(30.4 \%)$ & $46(29.5 \%)$ & $49(31.4 \%)$ & \\
\hline Noninvasive mechanical ventilation & $31(9.9 \%)$ & $15(9.6 \%)$ & $16(10.3 \%)$ & \\
\hline Invasive mechanical ventilation & $2(0.6 \%)$ & $0(0.0 \%)$ & $2(1.3 \%)$ & \\
\hline
\end{tabular}

Table 3 Risk factors associated with in-hospital death after PSM

\begin{tabular}{|c|c|c|c|c|}
\hline & \multicolumn{2}{|c|}{ Univariable } & \multicolumn{2}{|c|}{ Multivariable } \\
\hline & OR $(95 \% \mathrm{CI})$ & $P$ value & OR $(95 \% \mathrm{CI})$ & $P$ value \\
\hline 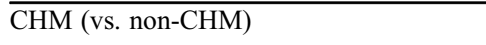 & $0.303(0.154,0.598)$ & $<0.001$ & $0.178(0.076,0.418)$ & $<0.001$ \\
\hline Female (vs. male) & $0.698(0.379,1.287)$ & 0.249 & & \\
\hline Age (year)* & $1.068(1.040,1.096)$ & $<0.001$ & $1.053(1.023,1.084)$ & $<0.001$ \\
\hline Severe cases (vs. critical cases) & $0.065(0.032,0.133)$ & $<0.001$ & $0.063(0.028,0.143)$ & $<0.001$ \\
\hline Comorbidity present (vs. not present) & $1.305(0.699,2.435)$ & 0.403 & & \\
\hline Imaging features present (vs. not present) & $0.724(0.258,2.031)$ & 0.539 & & \\
\hline \multicolumn{5}{|l|}{ Treatment (vs. non-treatment) } \\
\hline Antibiotics & $1.095(0.500,2.399)$ & 0.820 & & \\
\hline Antiviral treatment & $0.619(0.284,1.347)$ & 0.227 & & \\
\hline Chinese patent medicine & $0.780(0.418,1.455)$ & 0.435 & & \\
\hline
\end{tabular}

*Per 1 unit increase.

Given the lack of effective therapies for COVID-19, supportive care, including symptom control and complication prevention, remains the most commonly used treatment option [11]. In addition, glucocorticoid treatment is beneficial for specific patients [12]. Many antiviral drugs, including arbidol $[13,14]$ and remdesivir $[15,16]$, are recommended for the treatment of COVID-19, and some pharmacological studies also support the use of these drugs to inhibit SARS-CoV-2. However, the use of antiviral drugs in many clinical trials, including this study, is not a key factor affecting mortality. This phenomenon may be attributed to the fact that in patients with mild COVID-19 [16], antiviral drugs can reduce the clinical symptoms and promote the elimination of virus.
However, in patients with severe and critical COVID-19, antiviral therapy is only a basic treatment. Whether or not it is combined with organ failure is a more important risk factor; thus, the treatment used to improve the condition of patients may be more significant [11].

Although CHM has been applied to treat many patients with COVID-19, limited studies have confirmed its clinical effects on this disease [17]. In China, traditional Chinese medicine (TCM) has been widely used in the prevention and treatment of COVID-19, and some studies showed that CHM can reduce the antipyretic time in mild patients [18], shorten the average hospital stay, and improve the CT improvement rate [19]. However, the sample sizes of these studies were small, and limited clinical evidence was 
achieved to support the application of CHM in patients with COVID-19. In addition, studies examining the use of CHM in patients with severe or critical COVID-19 are lacking. This retrospective cohort study is the first to reveal that the use of CHM in patients with severe/critical COVID-19 can reduce mortality. Thus, TCM may be also an option in the treatment of patients with severe or critical COVID-19 in addition to antiviral and supportive therapies.

In TCM theory, the main principles include opening the inhibited lung energy and relieving cough, resolving dampness and detoxicating, and tonifying qi and strengthening middle warmer, focusing on improving patients' healthy qi and dispelling the evil factors to regulate the internal environment, enhance immunity, improve antiviral activity, inhibit inflammatory response, improve patient symptoms, prevent organ failure, and reduce mortality. The main herbs involved in this study are Mahuang, Kuxingren, Fuling, Baizhu, Huoxiang, Peilan, Huangqi, Dangshen, and so on. Pharmacological studies revealed that Mahuang [20], Kuxingren [21], Peilan [22], and Huangqi [23] can relieve bronchial mucosal edema; relax bronchial smooth muscle and eliminate phlegm; improve cough, dyspnea, and other symptoms; and protect the bronchial mucosa. Huoxiang [24] and Mahuang [25] have certain antiviral and anti-inflammatory effects. Huangqi [26,27], Dangshen [28,29], Baizhun [30], and Fuling $[31,32]$ can enhance immunity and improve failure of organs, such as the heart and the brain, caused by ischemia and hypoxia. We also discovered an effective CHM against COVID-19 by using the TCMATCOV platform in another study [33]. Results showed that the "multi-component and multi-target" characteristics of CHM act on COVID-19 disease-specific factors, including SOCS3, IL7, CXCI10, IL2, TNF, IL10, CCL2, and CCL3 directly or indirectly, and exert anti-inflammatory, antiviral and immunemodulating effects at multiple targets and levels.

Although this study has important clinical and research significance to guide the application of $\mathrm{CHM}$ in patients with severe/critical COVID-19, it still have some important limitations that need to be considered. First, this retrospective cohort study failed to include all baseline characteristics and patients' medical records before hospitalization, which possibly affected the authenticity of the study results. The baseline levels of all indicators between the groups were not completely balanced. The two groups showed significant differences in terms of sex, age, disease duration, medical history, antibiotics treatment, application of Chinese patent medicines, and oxygen therapy. PSM was performed in this study to maintain the baseline balance between the two groups. However, only the main variables were matched because of the sample size. Therefore, even after PSM, variables in comorbidities can still affect the study results. In addition, the sample size of the study after PSM decreased from 662 to 312, and the loss of a large amount of patient information may lead to selective bias. Therefore, a larger sample cohort study or a randomized controlled study is necessary in the future to improve the reliability of results.

\section{Acknowledgements}

This research was supported by the Special Project for Emergency of the Ministry of Science and Technology of China (No. 2020YFC0845000), the Traditional Chinese Medicine Special Project for COVID-19 Emergency of National Administration of Traditional Chinese Medicine (No. 2020ZYLCYJ04-1), and Special Project for Emergency of the National Administration of Tradition Chinese Medicine of China (No. 2020ZYLCYJ03-11). The funders had no role in the design and conduct of the study; collection, management, analysis, and interpretation of the data; preparation, review, or approval of the manuscript; and decision to submit the manuscript for publication.

\section{Compliance with ethics guidelines}

Guohua Chen, Wen Su, Jiayao Yang, Dan Luo, Ping Xia, Wen Jia, Xiuyang Li, Chuan Wang, Suping Lang, Qingbin Meng, Ying Zhang, Yuhe Ke, An Fan, Shuo Yang, Yujiao Zheng, Xuepeng Fan, Jie Qiao, Fengmei Lian, Li Wei, and Xiaolin Tong declare that they have no conflict of interest. This study was approved by the institutional ethics board of Wuhan Integrated TCM and Western Medicine Hospital (No. [2020]8) and was registered with chictr.org. cn (ChiCTR2000030719), and all procedures followed were in accordance with the ethical standards.

\section{References}

1. Sohrabi C, Alsafi Z, O'Neill N, Khan M, Kerwan A, Al-Jabir A, Iosifidis C, Agha R. World Health Organization declares global emergency: a review of the 2019 novel coronavirus (COVID-19). INT J SURG 2020; 76:71-76

2. Rajgor DD, Lee MH, Archuleta S, Bagdasarian N, Quek SC. The many estimates of the COVID-19 case fatality rate. Lancet Infect Dis 2020; 20(7): 776-777

3. World Health Organization. Coronavirus disease 2019 (COVID-19) situation report-69. 2020. https://www.who.int/docs/default-source/ coronaviruse/situation-reports/20200329-sitrep-69-covid-19.pdf? sfvrsn $=8 d 6620 \mathrm{fa} \_8$ (accessed March 20, 2020)

4. Yang X, Yu Y, Xu J, Shu H, Xia J, Liu H, Wu Y, Zhang L, Yu Z, Fang M, Yu T, Wang Y, Pan S, Zou X, Yuan S, Shang Y. Clinical course and outcomes of critically ill patients with SARS-CoV-2 pneumonia in Wuhan, China: a single-centered, retrospective, observational study. Lancet Respir Med 2020; 8(5): 475-481

5. Cao B, Wang Y, Wen D, Liu W, Wang J, Fan G, Ruan L, Song B, Cai Y, Wei M, Li X, Xia J, Chen N, Xiang J, Yu T, Bai T, Xie X, Zhang L, Li C, Yuan Y, Chen H, Li H, Huang H, Tu S, Gong F, Liu Y, Wei Y, Dong C, Zhou F, Gu X, Xu J, Liu Z, Zhang Y, Li H, Shang L, Wang K, Li K, Zhou X, Dong X, Qu Z, Lu S, Hu X, Ruan S, Luo S, Wu J, Peng L, Cheng F, Pan L, Zou J, Jia C, Wang J, Liu X, Wang S, Wu X, Ge Q, He J, Zhan H, Qiu F, Guo L, Huang C, Jaki 
T, Hayden FG, Horby PW, Zhang D, Wang C. A trial of lopinavirritonavir in adults hospitalized with severe COVID-19. N Engl J Med 2020; 382(19): 1787-1799

6. Zhang K. Is traditional Chinese medicine useful in the treatment of COVID-19? Am J Emerg Med 2020; [Epub ahead of print] doi: 10.1016/j.ajem.2020.03.046

7. National Health Commission of the People's Republic of China. Notice on the issuance of the fifth edition of the Diagnosis and Treatment Guideline for COVID-19 (Trial Version). 2020. http:// www.nhc.gov.cn/yzygj/s7653p/202002/3b09b894ac9b4204a79db5b8912d4440.shtml (in Chinese) (accessed February 5, 2020)

8. Zhou F, Yu T, Du R, Fan G, Liu Y, Liu Z, Xiang J, Wang Y, Song B, Gu X, Guan L, Wei Y, Li H, Wu X, Xu J, Tu S, Zhang Y, Chen H, Cao B. Clinical course and risk factors for mortality of adult inpatients with COVID-19 in Wuhan, China: a retrospective cohort study. Lancet 2020; 395(10229): 1054-1062

9. Wu C, Chen X, Cai Y, Xia J, Zhou X, Xu S, Huang H, Zhang L, Zhou X, Du C, Zhang Y, Song J, Wang S, Chao Y, Yang Z, Xu J, Zhou X, Chen D, Xiong W, Xu L, Zhou F, Jiang J, Bai C, Zheng J, Song Y. Risk factors associated with acute respiratory distress syndrome and death in patients with coronavirus disease 2019 pneumonia in Wuhan, China. JAMA Intern Med 2020; 180(7): 934

10. Wang D, Hu B, Hu C, Zhu F, Liu X, Zhang J, Wang B, Xiang H, Cheng Z, Xiong Y, Zhao Y, Li Y, Wang X, Peng Z. Clinical characteristics of 138 hospitalized patients with 2019 novel coronavirus-infected pneumonia in Wuhan, China. JAMA 2020; 323(11): 1061

11. Runfeng L, Yunlong H, Jicheng H, Weiqi P, Qinhai M, Yongxia S, Chufang L, Jin Z, Zhenhua J, Haiming J, Kui Z, Shuxiang H, Jun D, Xiaobo L, Xiaotao H, Lin W, Nanshan Z, Zifeng Y. Lianhuaqingwen exerts anti-viral and anti-inflammatory activity against novel coronavirus (SARS-CoV-2). Pharmacol Res 2020; 156: 104761

12. Zhang W, Zhao Y, Zhang F, Wang Q, Li T, Liu Z, Wang J, Qin Y, Zhang X, Yan X, Zeng X, Zhang S. The use of anti-inflammatory drugs in the treatment of people with severe coronavirus disease 2019 (COVID-19): the perspectives of clinical immunologists from China. Clin Immunol 2020; 214: 108393

13. Li G, De Clercq E. Therapeutic options for the 2019 novel coronavirus (2019-nCoV). Nat Rev Drug Discov 2020; 19(3): 149150

14. Deng L, Li C, Zeng Q, Liu X, Li X, Zhang H, Hong Z, Xia J. Arbidol combined with LPV/r versus LPV/r alone against coronavirus disease 2019: a retrospective cohort study. J Infect 2020; 81(1): e1-e5

15. Al-Tawfiq JA, Al-Homoud AH, Memish ZA. Remdesivir as a possible therapeutic option for the COVID-19. Travel Med Infect Dis 2020; 34: 101615

16. Wang M, Cao R, Zhang L, Yang X, Liu J, Xu M, Shi Z, Hu Z, Zhong W, Xiao G. Remdesivir and chloroquine effectively inhibit the recently emerged novel coronavirus $(2019-\mathrm{nCoV})$ in vitro. Cell Res 2020; 30(3): 269-271

17. Ren JL, Zhang AH, Wang XJ. Traditional Chinese medicine for COVID-19 treatment. Pharmacol Res 2020; 155: 104743

18. Ni L, Zhou L, Zhou M, Zhao J, Wang DW. Combination of western medicine and Chinese traditional patent medicine in treating a family case of COVID-19. Front Med 2020; 14(2): 210-214

19. Wang Z, Chen X, Lu Y, Chen F, Zhang W. Clinical characteristics and therapeutic procedure for four cases with 2019 novel coronavirus pneumonia receiving combined Chinese and Western medicine treatment. Biosci Trends 2020; 14(1): 64-68

20. Waldeck B, Widmark E. The interaction of ephedrine with $\beta$ adrenoceptors in tracheal, cardiac and skeletal muscles. Clin Exp Pharmacol Physiol 1985; 12(4): 439-442

21. Chang HK, Yang HY, Lee TH, Shin MC, Lee MH, Shin MS, Kim CJ, Kim OJ, Hong SP, Cho S. Armeniacae semen extract suppresses lipopolysaccharide-induced expressions of cyclooxygenase [correction of cycloosygenase]-2 and inducible nitric oxide synthase in mouse BV2 microglial cells. Biol Pharm Bull 2005; 28(3): 449454

22. Sun SM, Song YM, Liu J, Yu SR. Pharmacological effects of volatile compounds from Eupatorium fortunei Turcz. Nothwest Pharm J (Xi Bei Yao Xue Za Zhi) 1995; 10: 24-26 (in Chinese)

23. Shen HH, Wang K, Li W, Ying YH, Gao GX, Li XB, Huang HQ. Astragalus membranaceus prevents airway hyperreactivity in mice related to Th2 response inhibition. J Ethnopharmacol 2008; 116(2): 363-369

24. Li YC, Xian YF, Su ZR, Ip SP, Xie JH, Liao JB, Wu DW, Li CW, Chen JN, Lin ZX, Lai XP. Pogostone suppresses proinflammatory mediator production and protects against endotoxic shock in mice. $\mathrm{J}$ Ethnopharmacol 2014; 157: 212-221

25. Hyuga S, Hyuga M, Oshima N, Maruyama T, Kamakura H, Yamashita T, Yoshimura M, Amakura Y, Hakamatsuka T, Odaguchi H, Goda Y, Hanawa T. Ephedrine alkaloids-free Ephedra Herb extract: a safer alternative to ephedra with comparable analgesic, anticancer, and anti-influenza activities. J Nat Med 2016; 70(3): 571-583

26. Fu J, Wang Z, Huang L, Zheng S, Wang D, Chen S, Zhang H, Yang $\mathrm{S}$. Review of the botanical characteristics, phytochemistry, and pharmacology of Astragalus membranaceus (Huangqi). Phytother Res 2014; 28(9): 1275-1283

27. Liu D, Chen L, Zhao J, Cui K. Cardioprotection activity and mechanism of Astragalus polysaccharide in vivo and in vitro. Int $\mathrm{J}$ Biol Macromol 2018; 111: 947-952

28. Li Z, Zhu L, Zhang H, Yang J, Zhao J, Du D, Meng J, Yang F, Zhao Y, Sun J. Protective effect of a polysaccharide from stem of Codonopsis pilosula against renal ischemia/reperfusion injury in rats. Carbohydr Polym 2012; 90(4): 1739-1743

29. Liu C, Chen J, Li E, Fan Q, Wang D, Li P, Li X, Chen X, Qiu S, Gao $\mathrm{Z}$, Li H, Hu Y. The comparison of antioxidative and hepatoprotective activities of Codonopsis pilosula polysaccharide (CP) and sulfated CP. Int Immunopharmacol 2015; 24(2): 299-305

30. Lee JC, Lee KY, Son YO, Choi KC, Kim J, Kim SH, Chung GH, Jang YS. Stimulating effects on mouse splenocytes of glycoproteins from the herbal medicine Atractylodes macrocephala Koidz. Phytomedicine 2007; 14(6): 390-395

31. Sun Y. Biological activities and potential health benefits of polysaccharides from Poria cocos and their derivatives. Int J Biol Macromol 2014; 68: 131-134

32. Ríos JL. Chemical constituents and pharmacological properties of Poria cocos. Planta Med 2011; 77(7): 681-691

33. Wang C, Ming H, Jia W, Su W, Zhang LR, Luo D, Yang JY. Analysis on medication regularity and pharmacodynamic characteristics of traditional Chinese medicine treatment of 444 severe cases of COVID-19. China J Chin Materia Medica (Zhongguo Zhong Yao $\mathrm{Za}$ Zhi) 2020; [Epub ahead of print] doi:10.19540/j.cnki. cjcmm.20200427.501 (in Chinese) 